\title{
Problemas Éticos y Representaciones Sociales de la Evaluación en la Universidad
}

\section{Ethical Problems and Social Representations of Evaluation at University}

\author{
Nelly Patricia Bautista Cárdenas* \\ Universidad Cooperativa de Colombia
}

\begin{abstract}
Este artículo es producto de una investigación sobre la evaluación en educación superior desde el aspecto cultural a partir de las representaciones sociales. La motivación para la reflexión fue la sobrevaloración de la calificación frente al aprendizaje percibida en la población estudiantil. La primacía en la nota conduce a crear un vacío en el campo ético y cívico además de anular la función formativa de la evaluación en el curso del desarrollo de una carrera universitaria. Por eso, el objetivo de la investigación consistió en evidenciar la representación social de la evaluación como fin y no como medio en el contexto universitario, permitiendo así exhortar a las entidades universitarias a que promuevan acciones que neutralicen las conductas violentas o delictivas de quienes pretenden obtener calificaciones aprobatorias o certificaciones por encima de la lógica educativa y de los derechos humanos. La metodología utilizada es de orden cualitativo en el marco de las representaciones sociales, lo cual permitió relacionar los discursos constitucionales y las normas institucionales con el sentido común que construyen docentes y estudiantes ante el proceso evaluativo, encontrándose elementos subyacentes de poder, manipulación, temor, plagio y conceptos de la evaluación diferentes al que predican la teoría y la norma.
\end{abstract}

Palabras clave: Ética, Evaluación universitaria, Derechos humanos, Metodología de la evaluación, Representaciones sociales.

This article is the result of research about Evaluation in higher education from the cultural aspect seen from the Social Representations. The motivation for reflection was overvalued about the perceived qualifying in the student population. The primacy in note leads to create a vacuum in the ethical and civic field and will void the formative role of evaluation in the course of development of college. Therefore, the purpose of investigation is to show the social representation of the evaluation as an end and not a means, in the university context allowing institutions to urge the university to promote actions to neutralize violent or criminal behavior of those that seek passing grades or certifications over educational logic and human rights. The methodology used is qualitative in the framework of social representations, allowing relate the constitutional discourse and institutional rules with common sense that build teachers and students to the evaluation process, finding underlying elements of power, manipulation, fear, plagiarism and concepts of the different assessment to preaching theory and policy.

Keywords: Ethics, University evaluation, Human rights, Evaluation Methodology, Social representations.

\begin{tabular}{lll}
\hline *Contacto: nelly.Bautista@campusucc.edu.co & Recibido: & 22 de enero de 2016 \\
issn: 1989-0397 & $1^{\text {a }}$ Evaluación: & 24 de febrero de 2016 \\
www.rinace.net/riee/ & Aceptado: & 11 de marzo de 2016
\end{tabular}




\section{Introducción}

La evaluación forma parte de la historia del ser humano desde su nacimiento, lo que conduce a construcciones sociales que han hecho los actores de la educación en forma paralela a los enfoques teóricos y a las normas que le subyacen, reconociendo y moldeando las representaciones sociales que evidencia el mundo de la vida, complejo, contradictorio y subjetivo, que moldea el mundo normativo de la sociedad.

En este sentido, la investigación evidenció el aspecto ético de la evaluación en el contexto universitario con base en datos recogidos en la labor docente, donde se encontró que algunos estudiantes del programa de psicología de la Universidad Cooperativa de Colombia, al dar prioridad a la calificación sobre el aprendizaje y al Diploma sobre el profesionalismo, trasgreden los códigos éticos, morales y sociales que subyacen no solo a las normas institucionales sino también a los derechos humanos y los principios de dignidad humana, en cuyos cimientos filosóficos se sustenta el concepto de autonomía, derechos y libertades contenidos en la Carta Magna colombiana, cuyo disfrute implica la responsabilidad sobre los alcances de las acciones individuales y colectivas.

Los académicos entienden que la evaluación es la que diagnostica qué, cómo, por qué y cuándo enseñar, lo cual le da un lugar destacado a este acto formativo. En palabras de Arturo de la Orden (1981), "la evaluación, al prescribir realmente los objetivos de la educación, determina, en gran medida lo que los alumnos aprenden y cómo lo aprenden, lo que los profesores enseñan y cómo lo enseñan, los contenidos y los métodos" (p. 120). Es decir, la actividad educativa está en alto grado canalizada por la evaluación, lo que nos hace un llamado al aspecto ético que le subyace porque, al ser un elemento mediador en la educación, repercutirá en la formación integral del futuro profesional.

Se construyen normas generales desde el Ministerio de Educación e institucionales en cada centro educativo para delimitar parámetros claros y definidos en la labor educativa; se propende por una evaluación orientada al aprendizaje que se constituya en sí misma como un momento y una oportunidad para aprender. No obstante, el ser humano pone resistencia a los parámetros impuestos y complejiza su vida mediante la construcción de significados, símbolos y discursos que se entremezclan con las disposiciones oficiales, dando lugar a unas prácticas culturales que se hacen difíciles de reconocer en las acciones de los individuos. Es ahí donde ubicamos la tradición cultural expresada en las representaciones sociales que se entretejen con evaluación en la educación superior para reconocer el universo infinito que se instala en la subjetividad de los actores de la educación.

\section{Marco teórico}

\subsection{Concepto de evaluación de los aprendizajes}

La evaluación de los aprendizajes es un proceso complejo en el que intervienen elementos y factores variados como la institución, los profesores, los estudiantes, la enseñanza, los aprendizajes $\mathrm{y}$, en cada uno de ellos, todos los significados que se conjugan y que abre una brecha entre significados culturales y su concepto teórico.

Según Correa, Puerta y Restrepo (2002) "el término evaluación es una palabra elástica que se emplea para abarcar muchas clases de juicios” (p. 27). Se habla de evaluación de 
la ejecución de tareas, evaluación de la calidad de la educación, evaluación del clima organizacional. En ese sentido, por evaluación entienden algunos autores el control del éxito o fracaso de programas, proyectos o instituciones, para identificar y medir resultados. "Así, la evaluación es un esfuerzo por reconocer qué cambios se presentan durante y después de un programa de acción y qué parte de dichos cambios pueden atribuirse al programa" (Correa et al., 2002, p. 27).

A continuación, se presentan algunas de las definiciones que dan los autores más destacados que evidencian el cambio que ha tenido este tema en las últimas décadas.

Arturo de la Orden (1981): "Proceso de recogida y análisis de información relevante para describir cualquier faceta de la realidad educativa y formular un juicio sobre su adecuación a un patrón o criterio previamente establecido como base para la toma de decisiones" (citado por Chavarría y Borrel, 2003, p. 52).

Pedro Lafourcade (1984): "Etapa del proceso educacional que tiene por fin comprobar de modo sistemático en qué medida se han logrado los resultados previstos en los objetivos que se hubieren especificado con antelación” (citado por Ruiz, 2014, p. 2).

Robert Stake y Terry Denny definen el concepto de evaluación como el "descubrimiento de la naturaleza y el valor de algo", "una ayuda para descubrir el mérito y las deficiencias de un programa” (citado por Proppe, 1990, p. 325).

José Gimeno Sacristán (1996):

La evaluación hace referencia a cualquier proceso por medio del cual alguna o varias características de un alumno, de un grupo de estudiantes, de un ambiente educativo, de objetivos educativos, de materiales, profesores, programas, etc., reciben la atención del que evalúa, se analizan y se valoran sus características y condiciones en función de unos criterios o puntos de referencia para emitir un juicio que sea relevante para la educación. (p. 338)

Miguel Ángel Santos Guerra (1993, p. 6): "Proceso de diálogo, comprensión y mejora, como discusión y reflexión compartida de todos los que están implicados en la actividad evaluada".

Juan M. Álvarez (2001): La evaluación es una oportunidad para que quienes aprenden pongan en práctica "sus conocimientos y se sientan en la necesidad de defender sus ideas, sus razones, sus saberes". También es el momento en el que "afloren las dudas, las inseguridades, las ignorancias, si realmente hay intención de superarlas” (p. 13).

María José Rodríguez Conde (2005): "Conjunto de procesos sistemáticos de recogida, análisis e interpretación de información válida y fiable, que en comparación con una referencia o criterio nos permita llegar a una decisión que favorezca a la mejora del objeto evaluado" (citado por Ruiz, 2014, p. 2).

Mario de Miguel Díaz (2006): "Proceso planificado, integral y pertinente a las competencias que se desean alcanzar. Se desarrolla a través del planteamiento de tareas o desafíos que el estudiante debe resolver, necesitando para ello un conjunto integrado de conocimientos, destrezas y actitudes" (citado por Ruiz, 2014, p. 2).

Elena Cano (2008): "Proceso que utiliza diversidad de instrumentos e implica a diferentes agentes, con el propósito de proporcionar información sobre la progresión en el desarrollo de la competencia y sugerir caminos de mejora" (citado por Ruiz, 2014, p. 2). 
Tiburcio Moreno Olivos (2009): "La evaluación es el medio por el cual valoramos y conocemos una situación educativa, bien en su proceso, bien en un momento determinado, pudiendo conocer la efectividad de la actividad" (pp. 564-565).

Como se observa con las anteriores definiciones, el concepto de evaluación ha cambiado a través de los años. Inicialmente se entendía como medición del evaluado para modificarse progressivamente hacia la idea de una evaluación que mide a todos los implicados en esta actividad y cuya finalidad es servir de referencia para tomar decisiones y acciones de mejora.

\subsection{Estrategias de la evaluación}

Según Ronald Feo (2010), para el diseño de la estrategia de evaluación el profesor emplea de manera básica diferentes instrumentos que permitan recabar información. Esta información se puede alcanzar a través de técnicas evaluativas que conlleven la observación y análisis de los procesos o productos finales elaborados en las actividades. Es necesario resaltar que la estrategia de evaluación gira en torno a criterios evaluativos congruentes con la actividad evaluativa diseñada. Se utiliza una gran variedad de técnicas e instrumentos evaluativos como: observación, portafolios, mentefactos, entrevistas, encuestas, informes, estudios de caso, tareas, ensayos, exposiciones y pruebas escritas.

\subsection{Funciones de la evaluación}

La evaluación de los aprendizajes cumple diferentes funciones, entre ellas encontramos las definidas por Gimeno Sacristán (1996, p. 364):

Definición de los significados pedagógicos y sociales; funciones sociales; poder de control; funciones pedagógicas; creadora del ambiente escolar; diagnóstico; recurso para la individualización; afianzamiento del aprendizaje; función orientadora; base de pronósticos; ponderación del currículum y socialización profesional; funciones en la organización escolar; proyección psicológica; y apoyo de la investigación.

A continuación se explican estas funciones Gimeno Sacristán, 1988,:

$\checkmark$ Definición de los significados pedagógicos y sociales: "Los usos y resultados de la evaluación [...] generan realidad y sirven para pensar, hablar, investigar, planificar y hacer política educativa sobre la educación. La evaluación dota de contenido a la idea de 'excelencia escolar", que se utiliza para hablar de lo que resulta mejor o peor (pp. 364-365).

$\checkmark$ Funciones sociales: En una sociedad en la que "la acreditación del saber y de las formas de ser o comportarse, expresan la posesión de un capital cultural y de valores que se cotizan en la sociedad", los resultados de evaluación de los aprendizajes repercutirán en las "oportunidades de entrada al mercado laboral" ya que mide los niveles de competencia del evaluado (p. 365).

$\checkmark$ Poder de control: Se evidencia en "la actitud de algunos profesores al imponerlas, por la forma de realizarlas, la potestad de corregir respuestas interpretables" (p. 366), la posibilidad de discutir los resultados, se pueden convertir en un instrumento de dominación. El evaluador manifiesta los criterios sobre lo que es correcto o no.

$\checkmark$ Funciones pedagógicas: "La evaluación cumple un papel de regulación de los procesos de enseñanza y de aprendizaje, es decir, de reconocimiento de los cambios que deben introducirse en este proceso a fin de que cada estudiante aprenda de forma significativa" (p. 367). 
$\checkmark$ Creación del ambiente escolar: Es "la proyección que tiene la evaluación en las relaciones interpersonales" entre profesores y alumnos porque sus resultados sirven de referencia en la estructuración de las relaciones sociales. "Las normas $\mathrm{y}$ valores por las que funciona el grupo escolar tienen mucho que ver con el clima de evaluación" (p. 367).

$\checkmark$ Diagnóstico: Esta función es la que da a conocer el progreso y "el funcionamiento de los procesos de aprendizaje con el fin de intervenir en su mejora”. Se utiliza en el sentido de conocer al estudiante, sus saberes previos, sus condiciones personales, familiares y sociales; para obtener una perspectiva global de las personas en su propio contexto; conocimiento del curso para corregir y mejorar los procesos; proyección del estado final del alumno; y diagnóstico de las cualidades de los alumnos para la conformación de grupos (p. 367).

$\checkmark$ Recurso para la individualización: "La función diagnóstica de la evaluación permite la adaptación de la enseñanza a las condiciones del alumno y a su ritmo de progreso así como el tratamiento de dificultades particulares" (p. 369).

$\checkmark$ Afianzamiento del aprendizaje: La evaluación se encuentra dentro del proceso de seguir aprendiendo: Cuando la evaluación trata una lección concreta, activa en el estudiante su atención sobre aspectos importantes del contenido; si la evaluación es sobre módulos o cursos completos, activa la motivación del alumno hacia la asignatura. A largo plazo, se estimula la habilidad de retener y aplicar en contextos y formas diversas el material aprendido.

$\checkmark$ Función orientadora: Al detectar las cualidades y competencias en ciertas asignaturas, la evaluación sirve de guía para tomar decisiones apropiadas a la elección de tipos de estudios o la creación de materias optativas.

$\checkmark$ Base de pronósticos: Los resultados de las evaluaciones son referentes para crear imágenes de los alumnos en el medio escolar y en el exterior; sobre su valía académica o su 'excelencia'. Estas informaciones generan expectativas que condicionan la valoración de datos posteriores.

$\checkmark$ Ponderación del curriculum y socialización profesional: Las maneras de evaluar ponen de manifiesto los esquemas implícitos, la idea sobre conocimiento relevante y las ideas que se tienen sobre el "aprendizaje ideal". Estas reflexiones actúan como filtros en el desarrollo del currículum (p. 371).

$\checkmark$ Funciones en la organización escolar: La formación de "muchos alumnos durante un largo período de sus vidas implica enfrentarse a una heterogeneidad importante de condiciones, capacidades y posibilidades queriendo transmitirles aprendizajes muy diversos, reclama organizar alumnos y conocimientos", y es allí donde la evaluación refleja la complejidad de la organización del programa (p. 374).

$\checkmark$ Proyección psicológica: "La evaluación tiene repercusiones psicológicas en la motivación, en la modelación del autoconcepto personal, en las actitudes del alumno, en la generación de ansiedad y en la acentuación de conflictos o rasgos patológicos" (p. 376).

$\checkmark$ Apoyo de la investigación: Los resultados de la enseñanza y la evaluación son una fuente de información y conocimiento sobre cómo funciona el sistema educativo, siendo necesario considerar todo tipo de datos tanto cuantitativos como cualitativos para que realmente sean un apoyo fidedigno a la investigación (p. 378). 


\section{4. Ética en la Evaluación}

Introducir el tema de la ética en la acción evaluadora es abordar la moral como costumbre y norma instituida en el seno de la sociedad, con el fin de ubicar lo que desde la cultura se asume como vital para defender la óptima formación integral del individuo y del grupo de estudiantes dentro una sana convivencia. La evaluación entonces se torna un pretexto para discriminar entre lo bueno y lo malo, lo idóneo y lo insuficiente, lo legal y lo ilegal, por lo que se armoniza con la norma institucional y la jurídica ya que ambas buscan regular las acciones e interacciones de los actores sociales, que en este caso señala a quienes conforman la comunidad universitaria.

El ejercicio evaluativo se rige por tendencias nacionales e internacionales trascendiendo el acto inicial de promover a los estudiantes. En este sentido, desde el ámbito nacional la evaluación está atravesada por determinadas posturas políticas que exhiben su intervención en la normativa y en la práctica académica. Las tendencias internacionales, por su parte, fundamentan la formulación de dichas políticas imponiendo parámetros de competitividad a los profesionales, en el aspecto del saberhacer. En así que el fenómeno evaluativo se ve permeado por los discursos éticos y políticos que definen sus funciones.

Los discursos políticos tienen profundo impacto en la representación de la función ideal del proceso evaluativo y se desprenden de la misión y visión institucional, así como de estudios profundos en el tema. No obstante, es común encontrar que muchas veces los agentes evaluadores los infravaloran, centrándose fundamentalmente en lo técnico como forma de comprobar el saber profesional. Así se da prioridad al saber objetivo sobre el pensamiento ético, creativo y crítico.

Esta forma de entender la medición del conocimiento tiene una profunda carga ideológica y de poder que direcciona la manera como se seleccionan las técnicas, las herramientas, las formas y los procedimientos para desarrollar la evaluación, así como la valoración que se haga de los procesos y de los resultados. La evaluación de los aprendizajes no puede reducirse a la aplicación de instrumentos con el fin de recoger información, ya que esto coarta la responsabilidad ética y dificulta la formalización de metas claras que provean de significado las acciones educativas, restringiendo también la toma de decisiones.

Cuando el ejercicio evaluativo se mueve de manera sólida, apoyado por presupuestos teóricos y críticos que explicitan su sentido, se convierte en el seguimiento adecuado para dar cuerpo a la función formativa de la evaluación y dispone la apertura al pensamiento ético que redunda en compromiso profesional y social. No obstante, como lo demuestra la teoría y el método de las representaciones sociales (Jodelet, 1984), "el mundo no obra de acuerdo a los parámetros establecidos, ni a la razón sino a un complejo entramado que es lo que llamamos el mundo de la vida" (Bautista, 2015). La evaluación, como algo que está presente en la experiencia del ser humano desde el inicio de su existencia, en el contexto universitario también está cargada de sentido y no se limita únicamente a la selección, clasificación y orientación al grupo de estudiantes, sino que se ve permeada por acciones que en ocasiones se distancian del objetivo formativo, pasando a ser una mezcla de razón y sin razón evidenciada en acciones y emociones alejadas del ideal.

Es de recordar también en el aspecto ético que la academia reproduce y legitima las desigualdades sociales y, siendo la evaluación una herramienta que permite ordenar y clasificar a los estudiantes, se convierte en un parámetro decisivo de su futuro y su 
posición social (Murillo e Hidalgo, 2015). Por eso, como lo plantean estos investigadores, se debe seguir un enfoque de evaluación para justicia social que busque recuperar el valor ético del ejercicio evaluativo desde todos los actores de la educación.

\section{Representaciones sociales}

El concepto de representaciones sociales se sustenta teóricamente en tres corrientes básicas: la etnopsicología de Wundt, el interaccionismo simbólico de Mead y las representaciones colectivas de Emile Durkheim (Mora, 2002). La etnopsicología o psicología de los pueblos explica la evolución de la mente del hombre, la importancia del lenguaje, el pensamiento y la comunicación. Por su parte, la contribución del interaccionismo simbólico de George Mead fue su tesis de la intersubjetividad basada en lo que llamó la conversación interior desde tres interlocutores permanentes que eran el yo, el mí y el otro generalizado o realidad social, resaltándose el valor de la simbología en la comunicación. Por último, están los aportes de Emile Durkheim desde su teoría de las representaciones colectivas, donde sostiene que lo colectivo no puede ser reducido a lo individual imponiéndose parámetros sociales de pensamiento y acción en el individuo desde su nacimiento (Mora, 2002).

Estas influencias marcaron el desarrollo de la teoría que propuso Serge Moscovici sobre representaciones sociales. Para Moscovici (1979), estas son generadas en procesos de intercambios e interacciones, por lo cual decide desligarlas de la categoría preestablecida y estática que traía la visión clásica de Durkheim. Así, Moscovici sintetiza la construcción de representaciones sociales en los siguientes postulados (Araya, 2002, p. 26):

a) "Nuestro conocimiento, más que ser reproductor de algo preexistente, es producido de forma inmanente en relación con los objetos sociales que conocemos".

b) "La naturaleza de esa generación y construcción es social, esto es, que pasa por la comunicación y la interacción entre individuos, grupos e instituciones".

c) El lenguaje y la comunicación son "mecanismos en los que se transmite y crea la realidad, por una parte, y [...] marco en que la realidad adquiere sentido, por otra".

Con base en las anteriores premisas, Moscovici definió las representaciones sociales como una modalidad particular del conocimiento cuya función es la elaboración de los comportamientos y la comunicación entre los individuos. Dicho en diferentes palabras: quien conoce se pone dentro de aquello que conoce, en este caso, dentro de un ambiente social.

Por su parte, Denise Jodelet (1984), seguidora del pensamiento de Moscovici, sostiene que las representaciones sociales son: "imágenes que condensan un conjunto de significados; sistemas de referencia que nos permiten interpretar lo que nos sucede e incluso dar un sentido a lo inesperado; categorías que sirven para clasificar las circunstancias, los fenómenos y los individuos con quienes tenemos algo que ver" (p. $472)$.

En síntesis, las representaciones sociales constituyen sistemas cognitivos en los que es posible reconocer la presencia de estereotipos, opiniones, creencias, valores y normas que suelen tener una orientación actitudinal positiva o negativa. Se componen, "a su 
vez, como sistemas de códigos, valores, lógicas clasificatorias, principios interpretativos y orientadores de las prácticas, que definen la llamada conciencia colectiva, la cual se rige con fuerza normativa en tanto instituye los límites y las posibilidades de la forma en que las mujeres y los hombres actúan en el mundo" (Hoyos, 2013, p. 165).

\section{Método}

La investigación se desarrolló con un enfoque etnográfico desde un ángulo descriptivo e interpretativo, tomando como punto clave los significados construidos por la comunidad educativa para analizar las representaciones sociales en torno a la evaluación en el programa de Psicología de la Universidad Cooperativa de Colombia, sede Bogotá.

La etnografía es el estudio descriptivo de la cultura de una comunidad; el término es una denominación acuñada en el ámbito de los estudios antropológicos culturales. Para Anadón, "la etnografía es percibida como un enfoque que requiere inmersión directa del investigador en el medio estudiado con el fin de aprehender el "estilo de vida" de un grupo a partir de la descripción y la reconstrucción analítica e interpretativa de la cultura, de las formas de vida y de la estructura social del grupo estudiado" (citado por Bautista, 2011, p. 85).

La investigación etnográfica es un proceso de aproximación a una situación social, considerada de manera general en su propio contexto natural, método que permite lograr el objetivo fundamental de conocer las representaciones sociales que se han construido en torno a la evaluación de los aprendizajes en el contexto universitario, sobrepasando el discurso académico y accediendo a la comprensión empática del fenómeno objeto de estudio. Con este método, se pueden analizar y describir culturas y comunidades para acceder a las creencias y prácticas del grupo estudiado con el objeto de descubrir patrones y regularidades que surgen de la complejidad.

El referente de análisis fueron las representaciones sociales, utilizando técnicas como la asociación libre, para conseguir los significados espontáneos que emiten los estudiantes ante el término evaluación, y la observación, con el fin de examinar posturas, comentarios y actitudes ante el evento de diversos ejercicios evaluativos. Por último, el instrumento que más datos produjo fue la entrevista abierta que se realizó a estudiantes y docentes.

Al realizarse la investigación desde el programa de psicología, la población fue conformada por 60 estudiantes, 3 de cada semestre del diurno y 3 de cada semestre del nocturno, y 4 docentes del programa de psicología. Se incluyeron estudiantes de todos los semestres del diurno y del nocturno para conocer los criterios tanto de las personas que están iniciando la carrera como de los que ya han tenido algún proceso dentro de la misma. En cuanto a los docentes, se presentó la dificultad de contar con muy poca colaboración para la investigación.

\section{Resultados y discusión}

A continuación se exponen las concepciones de evaluación desde el punto de vista de los estudiantes y de los docentes donde se evidenciaron diferentes representaciones sociales derivadas del ejercicio evaluativo. Para llegar al reconocimiento de estas 
representaciones, se plantearon unas categorías de análisis respecto a la evaluación, a saber: concepto, método, función y ética en el ejercicio de la evaluación.

\subsection{Concepto}

Los conceptos que tienen docentes y estudiantes de la evaluación se expresan bien en representaciones sociales que las reducen a un ejercicio memorístico, o bien como un modo de aprendizaje.

\subsubsection{Evaluación como ejercicio de memorización}

La práctica evaluativa no es vista por la mayoría de los estudiantes como una forma de medir para replantear y mejorar el aprendizaje, sino como una actividad donde lo que se considera es la capacidad de memorización: "pienso que la evaluación como la tenemos en este momento, mide la cantidad de conceptos que se quedan en nuestra memoria por un determinado tiempo (...), la mayoría de la gente estudia para los exámenes (...) y después lo olvidan (...)". Este pensamiento demuestra que la evaluación no se considera un medio de valoración de una situación educativa, ni un proceso que permite llegar a una decisión favorable para el alumno.

Así, los estudiantes se sienten abordados con intención punitiva sin encontrar realmente la fortaleza de la evaluación como proceso de aprendizaje, que necesariamente incluye el enriquecimiento de la memoria, no en el sentido de memorización, sino como condición necesaria para que tenga lugar el aprendizaje; de hecho, aprender es guardar algo en la memoria. Las evaluaciones apuntan directamente a la capacidad de memoria; sin embargo, el concepto popular de la misma se manifiesta en la construcción de un rótulo negativo hacia la memoria reduciéndola a "recitar" puntualmente nombres, fechas y conceptos puntuales. No obstante, la memoria es la que va acumulando todo tipo de información nueva que conlleva la creación de competencias que requieren el uso de lo aprendido.

\subsubsection{Evaluación como aprendizaje}

El aprendizaje como resultado de la evaluación no es percibido por todos los estudiantes, ya que esta concientización se da a medida que la formación profesional va avanzando, como lo expresa una docente:

En los primeros semestres los estudiantes tienden a trabajar por la nota y no por el
aprendizaje; sin embargo, en (...) $5^{\circ}$ semestre en adelante, hay mayor tendencia a trabajar
por el aprendizaje. (...) han alcanzado una conciencia mayor sobre la profesión o porque
la asignatura les permite acercarse a una práctica educativa más aplicada. Por ende, la
evaluación en la vida académica de los estudiantes tiende a tener mayor impacto en cuanto
reconocen sus posibilidades, fortalezas y debilidades (...), además porque empiezan a
actuar en equipos reales de trabajo y esto apoya y enriquece su proceso.

La docente reconoce que el desarrollo de la asignatura tiene una incidencia importante en la construcción de una representación social favorable de la evaluación, asumiendo la responsabilidad del profesor en la concientización que tiene el alumno frente a este ejercicio.

En los semestres más avanzados, los estudiantes tienen más conciencia de la fortaleza del ejercicio como señaló una alumna de $10^{\circ}$ : "gracias a esos procesos de evaluación que continuamente se realizan, las cosas mejoran, es decir, se evalúa para mirar algo, intervenir en algo y se busca con ello generar una solución”. Los sujetos van llegando al verdadero concepto de la evaluación entendiéndola como una oportunidad para poner en práctica los conocimientos y como un proceso dinámico, abierto y útil para el 
evaluado y el evaluador, conceptos que se acercan a los expuestos en las perspectivas teóricas de la evaluación de aprendizajes. Así, la evaluación se asume como una forma de aprendizaje, ya que el estudiante es consciente de que al ejercer su profesión será evaluado permanentemente por el desempeño que tenga como psicólogo, tanto por las instituciones como por los mismos clientes y pacientes.

\subsection{Método en la evaluación}

Al indagar sobre los métodos utilizados en la evaluación de los aprendizajes se encuentra una representación social de variedad de técnicas e instrumentos utilizados en el ejercicio.

\subsubsection{Evaluación como variedad de instrumentos}

En el proceso evaluativo la mayoría de los estudiantes reconocen diferentes instrumentos que se utilizan en la cotidianidad del aprendizaje, tales como exámenes, parciales, talleres grupales, control de lectura, exposiciones; otros entienden por evaluación únicamente el examen parcial o final; y unos pocos asumen que evaluación es la que ellos hacen a los docentes.

Los docentes entrevistados manifiestan que en su práctica utilizan diferentes instrumentos de evaluación pero observan que existe falencia en la comprensión lectora de los estudiantes o no leen los enunciados, por lo que en muchas ocasiones no siguen las instrucciones para el desarrollo de los ejercicios. Un docente señala que "un estudiante pierde una evaluación por diversas razones como la falta de comprensión lectora y análisis, no seguir instrucciones y el bajo compromiso con la asignatura".

Los instrumentos utilizados en el programa de psicología están conforme a lo propuesto por Ronald Feo (2010), que resalta la conveniencia de utilizar diversos formatos que permitan recabar información apropiada a la temática en particular. En este sentido, se encuentran instrumentos de reflexión, memorísticos, investigativos, analíticos, descriptivos, de comprensión, de redacción, de observación, exposiciones, entre otros, los cuales son utilizados en su totalidad en la práctica evaluativa del programa de psicología de la Universidad Cooperativa.

Se reconocen, de forma general, dos grandes categorías de instrumentos, unos referentes al trabajo grupal y otros al trabajo individual. El trabajo grupal se evidencia en el desarrollo de talleres como respuesta al debate dado entre los integrantes del mismo; también predomina la oralidad en exposiciones, mesas redondas y análisis de casos. La prueba oral es privilegiada por muchos estudiantes porque pueden dar explicación desde su punto vista y ser retroalimentados simultáneamente, desplegándose una modalidad de coevaluación automática que fomenta el pensamiento crítico.

En la evaluación individual predomina la expresión escrita y los instrumentos memorísticos, impulsando el sentimiento de vigilancia y las emociones de ansiedad e inseguridad. El método escrito individual tiene un enorme impacto en los estudiantes llegándose a reducir el concepto de evaluación al instrumento de tipo memorístico y punitivo donde el estudiante se percibe como un sujeto pasivo que responde a un evaluador quien ha definido los parámetros de la prueba, el tiempo y la forma de calificar Es por esta práctica que se ha construido una representación social de la evaluación como formulario sin posibilidad de mostrar pensamiento creativo y crítico. 


\subsection{Función de la evaluación}

En la categoría de función de la evaluación se encontraron tres representaciones sociales: retroalimentación; calificación y requisito; e imposición.

\subsubsection{Evaluación y retroalimentación}

Los estudiantes exponen que algunos docentes hacen retroalimentación de los ejercicios, lo que convierte la evaluación en un método de aprendizaje y un modo en que el alumno puede observar las fortalezas y debilidades de su formación profesional: "me permite ver en lo que estoy fallando y en lo que tengo fortalezas"; así, un estudiante de $9^{\circ}$ semestre considera esta práctica importante para medir el conocimiento que ha adquirido en determinado tema, reconociendo la función diagnóstica de la evaluación.

No obstante, para otros la evaluación es una actividad normativa que los profesores aplican únicamente por cumplir un requisito y no explican la razón de la calificación registrada: "lo hacen como requisito para calificar". Un estudiante que piense de esta manera también puede considerar que realiza sus evaluaciones para cumplir el requisito. Así, se desconocen las funciones expuestas por Gimeno Sacristán (1996) referentes al rol pedagógico de la evaluación, a la proyección psicológica, al poder de control y a la organización educativa.

Como afirman Osorio y López Mendoza (2014), la evaluación es un proceso que supervisa la instrucción y la misma no se ubica en ningún momento instruccional en particular, pues se entiende que el monitoreo y la retroalimentación con fines instruccionales son constantes. Es así que la retroalimentación debe formar parte del interés formativo tanto del docente como del estudiante.

Algunos docentes afirman que, cuando el alumno recibe de vuelta un trabajo, lo que le interesa es la calificación obtenida y, en ese sentido, los comentarios que registra el profesor pierden interés. Así, el feedback tiene poca eficacia si el énfasis está puesto en la calificación, lo que desanima al docente, que no observa las bondades del ejercicio a la vez que el estudiante presume que avanzar implica dejar atrás lo que ya ha sido calificado.

\subsubsection{Evaluación como calificación y requisito}

Para muchos estudiantes la evaluación cumple el objetivo de otorgar una nota y se aleja de la actividad del aprendizaje, por lo que la calificación se torna el objetivo principal del ejercicio en el aula, ya que es la que permite que la persona sea promocionada a un curso siguiente. Así se entiende en estas palabras de un estudiante de $1^{\text {er }}$ semestre: "uno como estudiante puede utilizar unos métodos o lo que sea para alcanzar una nota para cumplir un requisito para ser evaluado sin necesidad de tener aprendizaje". Visto de este modo, la evaluación es una práctica vacía cuyo único objetivo es proferir calificaciones.

Como consecuencia de entender la evaluación como requisito, se piensa que este ejercicio se debe cambiar y no aplicarlo solo por norma y como medio de calificación, como lo manifiesta un estudiante de $5^{\circ}$ semestre: "yo pienso que, para mejorarlo, se debería evaluar en términos de procesos y en una dinámica discursiva, no limitarse a colocar una nota sino convertirla en un discurso, en donde el estudiante intervenga". Este es uno de los casos en que un estudiante demuestra la necesidad de cambiar las 
prácticas evaluativas para que no se limite a la obtención de un valor numérico que refleje lo aprendido, sino que represente los elementos que hay que reforzar en el aprendizaje del tema. El sujeto se acerca mediante su experiencia a entender la evaluación como un proceso de diálogo, comprensión y mejora, como discusión y reflexión compartida de todos los que están implicados en la actividad evaluada, conforme lo expone en sus investigaciones Santos Guerra (1993).

\subsubsection{Evaluación como imposición}

Los enfoques teóricos sobre evaluación de los aprendizajes van dirigidos al docente e implícitamente se le otorga un papel pasivo al estudiante, lo que ha creado una representación social de imposición o "molestia necesaria" para el alumnado. La pasividad del estudiante ante la formación que se desprende de la evaluación se observa de manera reiterada en el ámbito universitario. Así como el docente no siempre utiliza la evaluación como mecanismo de enseñanza, el estudiante tampoco se libera de una actitud reduccionista frente a este ejercicio y no vuelve sobre los resultados para mejorar su aprendizaje: "el estudiante se queda con lo que dice el docente, pero no se dirige a los libros, no practica en otros lados". Este comentario en $8^{\circ}$ semestre demuestra que algunas personas reconocen la pasividad de quienes están en formación al depender únicamente de los datos que reciben en las aulas, sin buscar ninguna forma de ampliar ese conocimiento.

La pasividad contradice un principio de la formación profesional y al tenor de la misión institucional de la Universidad Cooperativa referente a la reflexión teórica y práctica que permite acceder a la autonomía en el aprendizaje. Se predica la orientación hacia el pensamiento crítico y la búsqueda de transformaciones en los ámbitos social, cultural, educativo, tecnológico, científico y familiar. La evaluación en la educación superior se debe constituir en un eje de análisis del modelo positivista imperante, ya que pone en juego tanto las potencialidades del estudiante universitario como sus limitaciones, todo lo cual es una motivación para el razonamiento en aras a movilizar discursos y paradigmas estáticos y hegemónicos que coartan el dominio del propio comportamiento, de los pensamientos y de las emociones.

\section{4. Ética}

La categoría ética surgió especialmente de la observación y la técnica de asociación libre, lo que resaltó algunas características sociales que se han implantado como representaciones de modalidades para interactuar en el mundo. Aquí se encontró el imaginario social sobre el uso del poder, el plagio y la violencia como recurso.

\subsubsection{La evaluación como ejercicio de poder}

En el tema de la evaluación surge la representación social respecto al poder del docente tanto en el buen entendimiento del término, como en la utilización corrupta del mismo. Así mismo, el estudiante evidencia su propio manejo de poder cuando traslada su responsabilidad a la institución o al docente, cambia de opinión y de comportamiento según su conveniencia, invoca razones lógicas pero descontextualizadas para encubrir sus demandas, exige la perfección de los demás y exacerba los errores, no admite sus propios incumplimientos en la presentación de trabajos, se victimiza por su incapacidad de interesarse por el aprendizaje o amenaza de forma encubierta para que el docente acceda a subir la nota.

La victimización estuvo presente en la historia colombiana desde la Conquista y la Colonia, donde se violentaron los derechos de los nativos. Esta situación ha pasado a la 
memoria colectiva como un recurso estratégico que demuestra un manejo de poder del individuo donde se asume como sujeto pasivo, atemorizado, dependiente y sumiso ante un supuesto dominio total del otro. En muchas situaciones cotidianas, el victimista se disfraza de víctima, consciente o inconscientemente, simulando una agresión o menoscabo inexistente y responsabilizando erróneamente al entorno o a los demás.

La autovictimización es una actitud que consiste en considerarse como el afectado por ciertas situaciones específicas y siempre ser el doliente de las circunstancias. En esta postura el individuo es incapaz de reconocer el mérito ajeno y considera que las demás personas deben sentir una pena profunda y un dolor solidario, que si no es así demuestran poca humanidad. Es por eso que esta estrategia se utiliza para probar la bondad y empatía del docente mediante un ablandamiento en la exigencia de la prueba o el rompimiento de normas que rigen el trabajo evaluado y que fuera acordado previamente.

Es evidente que es más fácil ponerse en la posición de víctima cuando el individuo es culpable de su incumplimiento, demostrando incapacidad para medir sus acciones y calcular las consecuencias de las mismas, y prefiere buscar la solución del problema adjudicándoles la responsabilidad a otras personas. Cuando el estudiante le muestra a los distintos docentes lo mal que está, se imagina que esta actitud se impregna en ellos convirtiéndose en una estrategia apropiada para enfrentar fallas demostradas en el acto evaluativo.

Por su parte, el docente también ejerce su poder en el tema aquí tratado; ejerce su dominio sobre lo que evalúa y cómo lo evalúa, incluso sobre la posibilidad de convertir esa evaluación en ejercicio de aprendizaje, de ofrecer la oportunidad o no de mejorar la calificación del alumno. No obstante, en su discurso tiene la percepción de perder cada vez más el dominio sobre su actividad, sintiéndose acosado, descalificado y culpado por alumnos, por los mensajes de la institución y por la sociedad en general, respecto al comportamiento de la comunidad estudiantil como resultado de una supuesta labor deficiente. No obstante, existe una representación del poder del docente en la población de estudiantes, de los mismos docentes y de la sociedad en general en el manejo de la evaluación de los aprendizajes.

El manejo abusivo del poder por parte del docente y del estudiante redunda en indiferencia. El desánimo del docente unido a la apatía del estudiante convierte la práctica evaluativa en un proceso aséptico, desprovisto de poder fructífero y de contenido ideológico. La discusión sobre el tema es pobre aunque conlleva al discurso ético de una educación sin voluntad que es la que finalmente determina las técnicas y los procedimientos y el sentido que se le otorga a los mismos. Quienes ejercen el poder desde el deseo y no desde el beneficio del otro generan tensiones y conflictos que deconstruyen el componente formativo de la evaluación.

\subsubsection{El plagio en el ejercicio evaluativo}

En la cultura estudiantil es común recurrir al plagio por medio del cual el estudiante pretende obtener calificaciones aprobatorias sin esforzarse; de esta manera la evaluación se convierte en un elemento de vicio y corrupción ratificándose la supremacía del valor de la nota sobre el aprendizaje.

Un reconocido político dijo alguna vez "tenemos que reducir la corrupción a sus justas proporciones” (Rojas, 2011). Con estas palabras se reconoce que la corrupción es una expresión de los vicios culturales que se han aprendido en un país donde la violación de 
los derechos ha sido una constante a través de la historia, expresada en las acciones de las personas en diferentes espacios, incluyendo el medio académico donde se evidencia a través del plagio como una perpetuación de la cultura del vivo.

La facilidad para acceder a cualquier información globalizada se ha convertido en una tentación constante para las personas que encuentran allí todo tipo de datos y reflexiones ya elaboradas que son compatibles con el lema estudiantil del "menor esfuerzo", afianzando una tendencia a la corrupción en el evento de tener que realizar ejercicios tediosos que otrora fueran parte de la rutina estudiantil, pero en la actualidad son motivo de descontento porque el tiempo de las personas se ha copado con las ocupaciones que ha traído la implementación de las TIC en la vida cotidiana y en la interacción social.

Con estas acciones se muestra que en la práctica la evaluación se puede deformar hasta niveles no previstos en teorías y normas, lo que hace indispensable una revisión de las conductas que suceden alrededor de los diferentes actos humanos que nunca están totalmente ligados a lo estipulado por instituciones, leyes y preceptos sociales. Esto lleva el tema de la evaluación a ser analizado desde los principios de los derechos humanos y la seguridad.

\subsubsection{La violencia como recurso}

Dentro del estudio adelantado se encontró a dos estudiantes a quienes denominaremos HO quienes a lo largo de su carrera cometieron toda clase de infracciones y acciones temerarias contra docentes, compañeros de estudio, directivos y personal administrativo, lo que puso en evidencia la poca preparación que tiene la institución ante casos extremos de anomia y anarquía que llegan a alcanzar los límites de la delincuencia.

Los estudiantes HO luego de su primera clase de la carrera iniciaron un liderazgo negativo y desde ese momento mantuvieron una actitud de protesta por todo lo que estaba a su alrededor. Los docentes y directivos respondían solícitamente a las peticiones, pero en poco tiempo esa actitud de desaprobación se transformó en vanagloria con la consecuente exigencia temeraria e irrespetuosa a todo docente que les reprobara sus incumplimientos en asistencia, tareas y ejercicios evaluativos.

Con el protagonismo desarrollado por $\mathrm{HO}$, se fue mostrando ante el grupo de estudiantes el poder que tiene la agresividad y se fueron generalizando actitudes irreverentes entre un grupo de alumnos que no admitían la pérdida de ningún ejercicio evaluativo. Así se vio dentro del claustro universitario cómo se construía una representación social de la violencia como medio de solucionar las dificultades. La conducta belicosa elimina la función formativa de la evaluación colocando como fin último un certificado sin sustento de competencias. Lo que se juega en la anulación de las bondades del ejercicio evaluativo son los principios básicos de humanidad, de respeto y de reconocimiento a la labor docente, de la imagen institucional y de la ética profesional.

Los estudiantes HO durante su proceso protagonizaron toda clase de acciones violentas para conseguir las calificaciones aprobatorias en todas las asignaturas. Para completar el historial de conducta abusiva, se anexaron a un trabajo de grado que estaba adelantando otro estudiante $\mathrm{y}$, al tener total incapacidad de relación pacífica, terminaron en conflicto con el estudiante, el asesor y toda persona que tratara de aplicar las normas del reglamento. Finalmente, estos estudiantes se graduaron con el 
trabajo del compañero, quien dejó de asistir a la universidad por temor a ser atacado. Así fue que esta graduación se logró mediante múltiples conflictos y agresiones no solo de $\mathrm{HO}$ sino de sus familiares y amigos que llegaban al campus con amenazas y escándalos para que se les suspendieran los procesos disciplinarios que tenían en curso por diversos motivos.

En este caso se demostró que en algunos estudiantes predomina la creencia de que la violencia es un recurso que conlleva al fortalecimiento de su identidad social, por lo que exhiben actitudes desafiantes como la agresión y la violación de las normas, lo cual no puede verse como un caso aislado, pues al examinarlo desde el prisma de las representaciones sociales se observa que la situación ha visibilizado pensamientos propios del sentido común de una sociedad que ha integrado en sus saberes culturales la imposición y la temeridad como un camino para asegurar sus derechos, olvidando los límites que afectan en una escala mayor los derechos humanos del entorno social.

Los medios violentos de expresión fueron rechazados por la mayoría de los estudiantes, quienes expresaron su descontento en relación a que a los agresores se les admitían todas las faltas mientras que a quien se acogía a la norma se le exigía mayor esfuerzo. Los argumentos que expone la comunidad estudiantil no son otros que ratificar la representación social respecto al predominio de la fuerza, ya que nunca se propuso un apoyo por parte de la comunidad para que prevaleciera el respeto a los derechos humanos, el conducto regular y el seguimiento de la norma como medio de recuperar la estabilidad institucional.

Los estudiantes violentos saltan para sí mismos el derecho fundamental del "debido proceso", puesto que buscan obtener la calificación aprobatoria de forma inmediata y con argumentos no válidos que solo fortalecen la temeridad y la osadía. El marco legal coloca el tema de la evaluación del aprendizaje y el desempeño humano en un rol determinado por el contexto de la ética de mínimos, cuyo principal referente es la Declaración Universal de los Derechos Humanos divulgados por la ONU en 1948. Desde el mismo principio filosófico, ético y axiológico, la Constitución Política de Colombia de 1991 en su artículo 67 define la concepción del Estado sobre la educación así: "La educación es un derecho de la persona y un servicio público que tiene una función social (...). La educación formará al colombiano en el respeto a los derechos humanos, a la paz y a la democracia”. Este principio constitucional se viola en el evento de asumir actitudes violentas contra cualquier sujeto de la comunidad educativa.

El problema radica en no asumir una actitud autoevaluativa que permita entender la necesidad de fortalecer la estructura profesional, actitud que no cambia al momento de desarrollar su trabajo de grado, buscando plagiar otros textos o acudir a los "carteles de tesis" donde venden el informe de investigación que solicita el estudiante (Benavides, 2012). Predominan así unos imaginarios que cada vez toman más fuerza en la población universitaria como el "todo vale" o "la ley es para violarla".

El trabajo de grado es la evaluación más importante que permite validar las competencias profesionales del estudiante, pero al ser todo sujeto de negociación ha dado lugar a la conformación de una zona negra en el ámbito educativo que abre una brecha entre la evaluación formativa, la formación ciudadana y la aptitud profesional. 


\section{Conclusiones}

Es asumiendo y no negando la complejidad del mundo de la vida que se hace necesario hacer una permanente reflexión sobre el factor ético de la evaluación del aprendizaje para que no se desdibuje el proceso formativo bajo acciones que se van implementando como forma rápida de solución de problemas que necesariamente están siempre presentes en toda actividad humana.

El manejo inadecuado del poder del docente y de los estudiantes en el ejercicio evaluativo conlleva al fortalecimiento de la injusticia que se enraiza en unas prácticas culturales, de ahí que es indispensable hacer un llamado a la comunidad universitaria para que toda actividad evaluativa se sustente los principios de Justicia social expuestos por Murillo e Hidalgo (2015): poseer una cultura de lucha por la Justicia Social; fomentar del desarrollo integral de los estudiantes basado en el respeto; desarrollar procesos académicos justos; reconocer la educación como un proceso vinculado con la raza, el género, la cultura, la orientación sexual y la capacidad del estudiante; tener altas expectativas hacia los estudiantes; contar con un liderazgo democrático y distribuido; fomentar la colaboración con el entorno social; y favorecer el pensamiento crítico y democrático.

La evaluación siempre encierra un juicio de valor y debe atender a diversos criterios en la formación del estudiante, quien a partir de la misma observará el desarrollo de sus capacidades en el ser, en el saber, en el hacer y en el convivir, por lo que exige no solo una adecuada planeación sino también una retroalimentación y la oportunidad de aprender y corregir desde el concepto recibido.

En el proceso evaluativo confluyen pensamientos, sentimientos, impulsos, costumbres y simbologías que convierten el espacio regulado por normas y perspectivas teóricas en una dimensión confusa y llena de prácticas que se alejan del discurso oficial. La evaluación en la educación superior es un tema en el que interviene la cultura y no solo debe ser vista desde el juzgamiento al docente, como se ha concebido tradicionalmente y lo manifiesta a manera de crítica en una entrevista Manuel Gil: "el prejuicio radica en considerar que, si no la única, por lo menos sí la principalísima causa de los problemas de los problemas del aprendizaje en el país es consecuencia de la incapacidad, la flojera, e incluso el fenotipo del personal docente" (Ordorika, Gil, Rodríguez y Rueda, 2016). Esto lleva a perpetuar la ausencia del estudiante en la optimización de la actividad evaluativa y buscar soluciones limitadas y sin trascendencia.

Analizar la postura de los actores sociales más incidentes en el proceso evaluativo nos lleva a una instancia más amplia que es la institución universitária, a la que le corresponde traspasar el texto normativo para crear mecanismos que permitan mantener en el lugar que corresponda las acciones de docentes y estudiantes, especialmente cuando se observa la desviación de la norma.

La universidad tiene un compromiso frente a la sociedad de formar profesionales competentes para responder a las dinámicas del mundo, no solo contribuyendo a la construcción y difusión del conocimiento y apoyando el desarrollo competitivo del país, sino también buscando el mejoramiento de la calidad de vida de las comunidades (Universidad Cooperativa de Colombia). Por ello, en concordancia con lo expuesto por el docente investigador Ricardo Gaete-Quezada (2015), es "fundamental atender adecuadamente las demandas internas mediante el correcto uso del poder y autoridad, 
resolviendo conflictos y generando acuerdos internos necesarios para mantener la estabilidad institucional”. (p. 8).

Es importante que la Universidad se proponga transformar las representaciones sociales imperantes sobre la evaluación como requisito o como mecanismo de poder para que se la conciba siempre como autoevaluación, entendiendo que el concepto del sí mismo se fundamenta en los mensajes recibidos del otro. Como lo afirma George Mead (1972), la sociedad es interacción y "funciona como un equipo y no es la acción individual aislada la que se impone, sino la comunicación y permanente retroalimentación que el individuo recibe del grupo social” (p. 15), lo que se convierte en autoconcepto.

\section{Referencias}

Álvarez, J. (2001). Evaluar para aprender, examinar para excluir. Madrid: Morata.

Araya, S. (2002). Las representaciones sociales. Ejes teóricos para la discusión. San José de Costa Rica: FLACSO.

Bautista, N. (2011). Proceso de la investigación cualitativa. Bogotá: Manual Moderno.

Bautista, N. (2015, 27 de abril). Investigación cualitativa: Análisis de caso. Recuperado de https://prezi.com/vl1g2-vroan1/investigacion-cualitativa-analisis-de-caso/

Benavides, J. (2012, 2 de noviembre). ¿Cuánto cuesta una tesis? Recuperado de http://www.cartelurbano.com/content/precio-de-una-tesis\#.U_9B5_15Mn4

Correa, S., Puerta, A. y Restrepo, B. (2002). Investigación evaluativa. Bogotá: ICFES.

Feo, R. (2010). Oreintaciones básicas para el diseño de estrategias didácticas. Tendencias Pedagógicas, 16, 220-236.

Gaete-Quezada, R. (2015). El techo de cristal en las universidades estatales chilenas. Un análisis exploratorio. Revista Iberoamericana de Educación Superior, 6(17), 3-20.

Gimeno Sacristán, J. (1996). Comprender y transformar la enseñanza. Madrid: Morata.

Hoyos, C. (2013). Representaciones sociales en el adolescente sobre la norma y el delito. Advocatus, 21(21), 161-172.

Jodelet, D. (1984). La representación social: Fenómenos, concepto y teoría. En S. Moscovice (Comp.), Psicología social II: Pensamiento y vida social (pp. 469-493). Barcelona: Paidós.

Mead, G. (1972). Espíritu, persona y sociedad. Buenos Aires: Paidós.

Mora, M. (2002). La teoría de las representaciones sociales de Serge Moscovici. Athenea Digital, 2, 1-25.

Moreno Olivos, T. (2009). La evaluación del aprendizaje en la universidad: Tensiones, contradicciones y desafíos. Revista Mexicana de Investigación Educativa, 14(41), 563-591.

Moscovici, S. (1979 ). El psicoanálisis, su imagen y su público. Buenos Aires: Huemul.

Murillo, F. J. e Hidalgo, N. (2015). Enfoques fundamentantes de la Evaluación de Estudiantes para la Justicia Social. Revista Iberoamericana de Evaluación Educativa, 8(1), 43-61.

Orden, A. de la (1981). Evaluación del aprendizaje y la calidad de la educación. En V. García Hoz (Comp.), La calidad de la educación. Exigencias científicas y condicionamientos individuales y sociales (pp. 111-132). Madrid: CSIC. 
Organización de Naciones Unidas. (1948). Declaración Universal de Derechos Humanos.

Osorio, K. L. y López Mendoza, A. (2014). La retroalimentación formativa en el proceso de enseñanza-aprendizaje de estudiantes en edad preescolar. Revista Iberoamericana de Evaluación Educativa, 7(1), 13-30.

Proppe, O. (1990). La investigación de la evaluación como una forma de potenciar el desarrollo en las escuelas y el profesionalismo en los profesores. Revista de Educación, 293, 325-345.

Rojas, J. (2011). Izquierda en Colombia: ¿Destinada a la muerte política? El Tiempo, 1-3.

Ordorika, I., Gil, M., Rodríguez, R. y Rueda, M. (2016). Reforma educativa y evaluación docente: El debate. Perfiles Educativos, 38(151), 190-207.

Ruiz, Y. A. (2014). Evaluación del aprendizaje: Aproximación conceptual. Aula Magna, 2, 1-14.

Santos Guerra, M. Á. (1993). La evaluación: Un proceso de diálogo, comprensión y mejora. Investigación en la Escuela, 20, 23-35. 